\title{
TAGUNG
}

\section{Rechtsfragen und Rechtsfolgeprobleme der gemeinsamen Handelspolitik nach dem Vertrag von Lissabon}

\author{
Sophie Luise Beuttenmüller*
}

Nach einer Begrüßung durch den Vorsitzenden des Arbeitskreises Europäische Integration e.V. Peter-Christian Müller-Graff führte Christoph Herrmann in das Tagungsthema ein. Bei der Tagung waren sowohl Vertreter der Wissenschaft als auch aus der anwaltlichen Praxis, aus dem Bundeswirtschaftsministerium, dem Auswärtigen Amt und den entsprechenden österreichischen Bundesministerien sowie auch Mitglieder des Europäischen Parlaments und der Europäischen Kommission zugegen. Diese Mischung der Teilnehmer spiegelt die Vielzahl der in der Praxis von der Neuordnung der Außenhandelskompetenzen betroffenen Ressorts wider. Die sich an die einzelnen Panels anschließenden Diskussionen wurden auch intensiv und kontrovers geführt. Es herrschte bei den Vortragenden und den Teilnehmern die überwiegende Auffassung vor, dass Art. 207 AEUV $^{1}$ der Europäischen Union fortan die ausschließliche Kompetenz für ausländische Direktinvestitionen und auch für jegliche von den WTO-Abkommen ${ }^{2}$ erfasste Materie zuweist.

Föderale Handelspolitik im europäischen Verfassungsverbund

Der Vorsitzende des ersten Panels Christian Tietje führte in das Thema ein, indem er darauf hinwies, dass bereits der Titel des Panels zwei Provokationen in sich trage. Denn die Worte „föderal“ und „Verfassungsverbund“

\section{Die gemeinsame Handelspolitik der Europäischen Union ,nach Lissabon“6}

Mit Unterstützung der Europäischen Kommission und Cleary Gottlieb Steen \& Hamilton, Brüssel

Berlin, 28. September 2010

\section{Wissenschaftliche Leitung}

Prof. Dr. Marc BUNGENBERG, LL.M, Universität Siegen

Prof. Dr. Christoph HERRMANN, LL.M., Universität Passau

\section{Begrüßung}

Prof. Dr. Dres. h.c. Peter-Christian MÜLLERGRAFF, Vorstandsmitglied des Arbeitskreises Europäische Integration e.V.; Universität Heidelberg

Einführung in das Tagungsthema Prof. Dr. Christoph HERRMANN, LL.M., Universität Passau

Föderale Handelspolitik im europäischen Verfassungsverbund Vorsitz: Prof. Dr. Christian TIETJE, LL.M., Martin-Luther-Universität Halle-Wittenberg

Ausschließlichkeit der Unionskompetenz in der GHP und die zukünftige Rolle der Mitgliedstaaten in der WTO

Prof. Dr. Michael HAHN, Universität Lausanne

\footnotetext{
* Sophie Luise Beuttenmüller, Wissenschaftliche Mitarbeiterin am Lehrstuhl für Öffentliches Recht und Europarecht Prof. Dr. Rudolf Streinz; Promotionsstipendiatin am Max-Planck-Institut für Immaterialgüter- und Wettbewerbsrecht, München.

1 Vertrag über die Arbeitsweise der Europäischen Union, in: Amtsblatt der EU, Nr. C 83 vom 30. März 2010, S. 47-199.

2 Die Abkürzung WTO steht für World Trade Organization.
} 
würden nicht von allen Ansichten uneingeschränkt in Zusammenhang mit der Europäischen Union gebracht. Damit spielte er auf die Finalitätsdebatte an.

Michael Hahn referierte sodann zur Ausschließlichkeit der Unionskompetenz in der gemeinsamen Handelspolitik (GHP) und zur zukünftigen Rolle der EU-Mitgliedstaaten in der WTO. Hahn skizzierte zunächst die historische Entwicklung der Zuständigkeitsverteilung von Union und Mitgliedstaaten für die Materien der WTO-Abkommen. Er führte an, dass das WTO-Übereinkommen im Jahr 1994 nach über dreißigjähriger Erfahrung aller Vertragsparteien des General Agreement on Tariffs and Trade (GATT) mit dem eigentümlichen Nebeneinander von Europäischer Gemeinschaft und Mitgliedstaaten geschlossen wurde. Bis zu einem Gutachten des Europäischen Gerichtshofs $(\mathrm{EuGH}),{ }^{3}$ das anlässlich eines Streits der Europäischen Gemeinschaft und ihrer Mitgliedstaaten um die Abschlusszuständigkeit eingeholt wurde, war von einer ausschließlichen Zuständigkeit der Gemeinschaft für die WTO-Materien auszugehen. Die neue Unionskompetenz führe nunmehr zu einer Rückkehr zu dieser damals bestehenden Kompetenzverteilung, da sie über Art. 207 Abs. 1 und Art. 3 Abs. 1 lit. e) AEUV der Union die ausschließliche Zuständigkeit aller derzeit in der WTO behandelten Materien einräume. Da jedoch das Welthandelsrecht eine dynamische Materie sei, verbliebe die Möglichkeit, dass innerhalb der WTO auch künftig Regelungsbereiche aufgenommen würden, die nicht von der derzeitigen primärrechtlichen Unionskompetenz abgedeckt seien. Hahn legte dar, wie sich der künftige Status der Mitgliedstaaten in der WTO nach Kompetenzverlagerung auf die Union aus Sicht des WTO-Rechts und aus Sicht des Unionsrechts darstellt. Das WTO-Recht enthalte keine Regelungen, die vorsehen, was im Falle einer Aufgabe der Zuständigkeitsbefugnisse einzel-
Gemeinsame Handelspolitik und deutsches Verfassungsrecht: Die Lissabon-Entscheidung des BVerfG und die verfassungsrechtlichen Grenzen einer Weiterentwicklung des europäischen Außenwirtschaftsrechts Dr. Jörg Philipp TERHECHTE, Universität Hamburg

Kommentar: Prof. Dr. Wolfgang WEIß, Deutsche Hochschule für Verwaltungswissenschaften Speyer

Die Rechtsordnung der Handelspolitik: Institutioneller und normativer Zuschnitt Vorsitz: Dr. Christian PITSCHAS, LL.M., Kanzlei Bernzen Sonntag, Berlin

Die zukünftige Rolle des Europäischen Parlaments in der gemeinsamen Handelspolitik Prof. Dr. Markus KRAJEWSKI, Universität Erlangen-Nürnberg

Die neue normative Struktur der autonomen Handelspolitik: Sekundärrechtsabhängigkeit der GHP?

Dr. Till MÜLLER-IBOLD, LL.M., Kanzlei Cleary Gottlieb Steen \& Hamilton, Brüssel

Kommentar: Dr. Godelieve QUISTHOUDTROWOHL, Mitglied des Europäischen Parlaments

Die gemeinsame Handelspolitik als Teil des auswärtigen Handelns der Union Vorsitz: Prof. Dr. Horst Günter KRENZLER, Ludwig-Maximilians-Universität München

Die gemeinsame Handelspolitik im Einflussbereich von Kommission, Rat, Hohem Vertreter und Europäischem Auswärtigem Dienst Prof. Dr. Hans-Georg DEDERER, Universität Passau

Die (Gemeinwohl-)Zielbindung der gemeinsamen Handelspolitik

Prof. Dr. Christoph VEDDER, Universität Augsburg

Die gemeinsame Handelspolitik als unionale Investitions(schutz)politik Vorsitz: Dr. Christina KNAHR, MPA, Universität Wien

3 Europäischer Gerichtshof: Gutachten des Gerichtshofes vom 15. November 1994 - Zuständigkeit der Gemeinschaft für den Abschluss völkerrechtlicher Abkommen auf dem Gebiet der Dienstleistungen und des Schutzes des geistigen Eigentums - Verfahren des Artikels 228 Absatz 6 EG-Vertrag. Gutachten 1/94, Slg. 1994 I, S. 5267. 
ner WTO-Mitgliedstaaten passieren sollte. Daraus schloss Hahn, dass ein Ausschluss oder Wegfall der Mitgliedschaft auch bei Fehlen oder künftigem Wegfall jeglicher Außenhandelskompetenzen der Mitgliedstaaten nicht gewollt war. Aus Sicht des Unionsrechts sei keine Rechtsgrundlage ersichtlich, aufgrund derer die Union von ihren Mitgliedstaaten verlangen könne, aus der WTO auszuscheiden. Solange die Mitgliedstaaten das Ihrige unternehmen würden, um eine Einheitlichkeit der Außenvertretung sicherzustellen, könne auch von Art. 351 Abs. 2 AEUV (ana$\log$ ) kein Gebrauch gemacht werden.

Sodann analysierte Jörg Philipp Terhechte die Aussagen des Bundesverfassungsgerichts (BVerfG) in der Lissabon-Entscheidung ${ }^{4} \mathrm{zu}$ der Neufassung der Zuständigkeitsverteilung zwischen Union und Mitgliedstaaten in der Außenhandelspolitik. Es falle ins Auge, mit welcher Ausführlichkeit das BVerfG sich zu diesem Thema geäußert habe. Auch stelle das BVerfG eine eigene Interpretation des Begriffs ,ausländische Direktinvestitionen“ vor. Dies sei umso erstaunlicher wenn man bedenke, dass nach Art. 19 EUV $^{5}$ der EuGH und nicht das BVerfG die alleinige Zuständigkeit habe, den Umfang und die Auswirkungen des Art. 207 AEUV zu bewerten. Dabei zeichnete er das Bild, dass das BVerfG mit seinen Aussagen am ,offenen Herzen der demokratischen Gesellschaft operiere“. Das BVerfG führte aus, dass die Union die Mitgliedstaaten trotz deren Kompetenzverlustes nicht zum Austritt aus der WTO zwingen könne. Dieser Aussage stimmte Terhechte uneingeschränkt zu. Kritisch würdigte er jedoch die Annahme des BVerfG, dass die Union die Pflicht habe, die bisherigen bilateralen Investitionsschutzabkommen der Mitgliedstaaten zu genehmigen. Eine solche Genehmigungspflicht ergebe sich weder aus dem Unionsprimärrecht noch aus den vom BVerfG diesbezüglich zitierten Sekundärrechtsakten des Rates.

\section{Bedeutung und Ziele des internationalen} Investitionsschutzrechtes

Dr. Markus BURGSTALLER, Sozietät Hogan Lovells, London

Der (primär)rechtliche Rahmen einer EUInvestitionsschutzpolitik

Dr. Steffen HINDELANG, LL.M., HumboldtUniversität zu Berlin

Zukünftige Rolle der Mitgliedstaaten in der EU-Investitionsschutzpolitik

Dr. Nils WEITH, Bundesministerium für Wirtschaft und Technologie, Berlin

Der Weg seit Lissabon - Bewertung der politischen Ansätze von EU und Mitgliedstaaten JunProf. Dr. Jörn GRIEBEL, D.E.S., Universität zu Köln

Kommentar: Prof. Dr. Andreas R. ZIEGLER, LL.M., Universität Lausanne

\section{Schlussworte}

Prof. Dr. Marc BUNGENBERG, LL.M., Universität Siegen

Der Kommentator Wolfgang Wei $\beta$ wies in der Frage der Rolle der Mitgliedstaaten in der WTO und auch bezüglich der rechtlichen $\mathrm{Zu}$ kunft der „Alt-Verträge“ der Mitgliedstaaten ausdrücklich darauf hin, dass die Mitgliedstaaten mit den Verträgen Verpflichtungen eingegangen seien, deren Bruch auch zur völkerrechtlichen Haftung der Mitgliedstaaten führen könne. Dies sei in der Diskussion der Rechtsfolgen der Zuständigkeitsverlagerung auf die Union nicht außer Acht zu lassen. Darüber hinaus stellte Weiß seine Interpretation zur Schrankenregelung des Art. 207 Abs. 6 AEUV vor. Er interpretierte diesen Absatz nicht als eine Beschränkung der Binnenkompetenz, sondern als eine auf den Umfang der Binnenkompetenzen reduzierte Beschränkung der Außenkompetenz. Wenn man Art. 207 Abs. 6 AEUV so lese, dann ergeben sich durchaus auch noch Bereiche des Welthan-

4 BVerfG: Verbundene Rechtssachen 2 BvE 2/08, 2 BvE 5/08, 2 BvR 1010/08, 2 BvR 1022/08, 2 BvR 1259/08, 2 BvR 182/09 vom 30. Juni 2009.

5 Vertrag über die Europäische Union, in: Amtsblatt der EU, Nr. C 83 vom 30. März 2010, S. 13-45. 
delsrechts, in denen die Union gerade nicht die ausschließliche Kompetenz habe.

Die Rechtsordnung der Handelspolitik: institutioneller und normativer Zuschnitt

Zunächst führte Markus Krajewski in die Frage ein, in welchem Zusammenhang Parlamente und Außenbeziehungen generell stehen und stellte eine traditionelle Dominanz der Exekutive in diesem Bereich fest. Diese könne auf John Locke zurückgeführt werden. Die Rolle der Parlamente sei auf die Zustimmung zu Außenhandelsverträgen begrenzt. Unter Verweis auf das Urteil des BVerfG zu den AWACS-Einsätzen ${ }^{6}$ stellte er heraus, dass dies auch für den Deutschen Bundestag gelte. Damit konstatierte er eine generell reduzierte demokratische Legitimation völkerrechtlicher Verträge im Vergleich zu innerstaatlichem Recht. Dem gegenüber stehe die geänderte Rolle des Europäischen Parlaments, das fortan weitreichende Mitentscheidungsrechte in der Europäischen Außenhandelspolitik erhält. ${ }^{7}$ So entscheiden künftig Rat und Parlament gemeinsam über den Rahmen der GHP, in dem sie im ordentlichen Gesetzgebungsverfahren nach Art. 207 Abs. 2 AEUV den Rahmen der GHP festlegen. Auch in der vertraglichen Handelspolitik ist fortan eine Zustimmung des Europäischen Parlaments erforderlich. ${ }^{8}$ Krajewski stellte vor, welche Auswirkungen dieser Kompetenzzuwachs auf das inter- und das intra-institutionelle Gefüge haben könne. Er stellte insbesondere eine gestiegene Bedeutung des Ausschusses für Internationalen Handel (INTA) des Europäischen Parlaments sowie einen Bedeutungs- zuwachs des Europäischen Parlaments auch aus internationaler Sicht zur Diskussion. Abschließend stellte er eine künftige Politisierung und Zielpluralität der GHP durch den Reformvertrag von Lissabon fest.

Im Anschluss ging Till Müller-Ibold insbesondere auf die Verfahren zum Erlass von Einzelfallmaßnahmen auf der Grundlage von Rahmenverordnungen nach Art. 207 Abs. 2 AEUV ein. In der Praxis folge auf den Vertragsschluss in vielen Fällen der Erlass von Durchführungsverordnungen, die es den Institutionen ermöglichen, zum einen die von der Europäischen Union einzunehmenden Positionen im institutionellen Gefüge des Vertrags zu bestimmen und zum anderen Maßnahmen im Einzelfall $\mathrm{zu}$ erlassen. Ein Beispiel für Letzteres sei die Verordnung (EWG) Nr. 2837/72 des Rates vom 19. Dezember 1972 über die im Abkommen zwischen der Europäischen Wirtschaftsgemeinschaft und der Republik Österreich vorgesehenen Schutzmaßnahmen. ${ }^{9}$ Die bisherige Grundlage für autonome Maßnahmen war Artikel 133 Abs. 2 EGV. ${ }^{10}$ Diese Norm galt als Rechtsgrundlage für Grundverordnungen zur abstrakten Regelung von handelspolitischen Maßnahmen und gleichzeitig als Grundlage für den Erlass von Einzelfallmaßnahmen, die von der Kommission vorgeschlagen und vom Rat einseitig erlassen wurden. Grundverordnungen seien dann möglich, wenn eine typisierbare Grundsituation geregelt werden soll, wie zum Beispiel bei der Einfuhrfreiheit und Schutzmaßnahmen ${ }^{11}$ oder beim Schutz vor Protektionismus in Drittstaaten. ${ }^{12}$ Sofern dies möglich war, durften auf der Grundlage des

6 Die Abkürzung AWACS steht für Airborne Early Warning and Control System.

7 Elmar Brok: Die neue Macht des Europäischen Parlaments nach ,Lissabon' im Bereich der gemeinsamen Handelspolitik, in: integration 3/2010, S. 209-223.

8 Vgl. Art. 207 Abs. 3 i.V.m. Art. 218 Abs. 6 UAbs. 2 lit. a) v) AEUV.

9 Amtsblatt der EG, Nr. L 300 vom 31. Dezember 1972, S. 94-95.

10 Vertrag zur Gründung der Europäischen Gemeinschaft, in: Amtsblatt der EU, Nr. C 321 E vom 29. Dezember 2006, S. 37-331.

11 Verordnung (EG) Nr. 260/2009 des Rates vom 26. Februar 2009 über die gemeinsame Einfuhrregelung, in: Amtsblatt der EU, Nr. L 84 vom 31. März 2009, S. 1-17.

12 Verordnung (EG) Nr. 3286/94 des Rates vom 22. Dezember 1994 zur Festlegung der Verfahren der Gemeinschaft im Bereich der gemeinsamen Handelspolitik zur Ausübung der Rechte der Gemeinschaft nach internationalen Handelsregeln, insbesondere den im Rahmen der Welthandelsorganisation vereinbarten Regeln, in: Amtsblatt der EU, Nr. L 349 vom 31. Dezember 1994, S. 71-78. 
EGV keine Einzelfallmaßnahmen erlassen werden. ${ }^{13}$ Aus der neuen Formulierung des Art. 207 Abs. 2 AEUV (,Rahmen für die Umsetzung") lasse sich nicht erkennen, ob darauf auch Einzelfallmaßnahmen gestützt werden können, ob also das Parlament zwingend an Einzelfallmaßnahmen zu beteiligen ist. Denkbar könnte auch eine Zuständigkeit nach der Lehre der ,implied powers' sein. Fraglich bleibt laut Müller-Ibold jedoch, auf welche Rechtsgrundlage Umsetzungsbefugnisse der Kommission gestützt werden können. In Betracht kommen hierfür entweder die delegierten Rechtsakte nach Art. 290 AEUV oder die Durchführungsrechtsakte nach Art. 291 AEUV. Letztendlich wird nach Müller-Ibold aber ein neuer Komitologiebeschluss hierüber nähere Aufschlüsse geben. ${ }^{14}$

Die Kommentatorin Godelieve QuisthoudtRowohl unterstrich das Ziel des Vertrags von Lissabon, mehr Transparenz in die EU-Institutionen zu bringen. Im Laufe der Zeit habe der Rat mehr und mehr Befugnisse an sich gezogen. Durch den neuen ständigen Präsidenten des Europäischen Rates würde eine absolute Konkurrenz zwischen Rat und Kommission begründet, die so vom Vertrag nicht vorgesehen war. Diese Situation spiegele sich auch in der GHP wieder. Das Europäische Parlament begrüße seinen Kompetenzzuwachs insgesamt und insbesondere auch im Rahmen der GHP. Aus Sicht des Europäischen Parlaments sei ein Mitspracherecht auch in der GHP ,ganz normal“" und man wundere sich, dass Deutschland als wichtigste und größte Exportnation der Europäischen Union so wenig Außenpolitik betreibe und der Bundestag so wenig inhaltliche Mitspracherechte in dieser Hinsicht habe. Zwar sei die GHP eine grundsätzliche Domäne der Exekutive und solle dies auch bleiben, aber es sei eine ausreichende Kontrolle der Exekutive durch die Parlamente erforderlich. Das neue Zustimmungs- erfordernis des Europäischen Parlaments zu den wirtschaftsvölkerrechtlichen Abkommen der Union könne in dieser Hinsicht als scharfes Schwert eingesetzt werden. QuisthoudtRowohl machte deutlich, dass das Europäische Parlament seine neuen Machtbefugnisse auch einsetzen und ein Abkommen gegebenenfalls ablehnen werde, wie sich bereits im Fall des SWIFT-Abkommens ${ }^{15}$ gezeigt habe. Darüber hinaus nannte sie weitere Möglichkeiten der Einflussnahme des Europäischen Parlaments auch außerhalb der GHP, wie beispielsweise das Haushaltsrecht des Parlaments. Eine Politisierung der GHP sah auch Quisthoudt-Rowohl. Jedoch unterstrich sie, dass dies keineswegs eine neue Entwicklung sei, vielmehr wären Bestrebungen, die GHP mit anderen Zielen, wie der Demokratisierung und der Achtung der Menschenrechte oder der Nachhaltigkeit, zu verbinden, bereits in den vergangenen Jahren verfolgt worden. Quisthoudt-Rowohl wies in diesem Zusammenhang auf die interparlamentarische Versammlung in der WTO hin, die schon lange auch andere als nur wirtschaftlich orientierte Ziele in den Handelsabkommen befürwortet. Mit der neuen Machtfülle des Europäischen Parlaments wachse dessen Bedeutung auch im Ausland, dies könne bereits durch eine Zunahme an Lobbyarbeit direkt bei den Abgeordneten deutlich festgestellt werden.

Die gemeinsame Handelspolitik als Teil des auswärtigen Handelns der Union

Hans-Georg Dederer ging in seinem Vortrag davon aus, dass es der Union im Konzert der Weltwirtschaftsmächte nur dann gelingen werde, als bestimmender Akteur aufzutreten, wenn sie hierfür im notwendigen Umfang auch organisatorisch-institutionell gerüstet sei. Dederer setzte sich intensiv mit den verschiedenen Funktionen des neuen Amtes des Hohen Vertreters der Union für die Außen-

13 Vgl. Europäischer Gerichtshof: Rs. 113/77 (NTN Toyo Bearing/Council), Slg. 1979, S. 1185.

14 Auf den Parlamentsbeschluss vom 16.12.2010 zur neuen Komitologieverordnung wird hingewiesen. Vgl. Europäisches Parlament: Kontrolle durch die Mitgliedstaaten der Wahrnehmung der Durchführungsbefugnisse durch die Kommission, P7_TA-PROV(2010)0488.

15 Die Abkürzung SWIFT steht für Society for Worldwide Interbank Financial Telecommunication. 
und Sicherheitspolitik auseinander, der gleichzeitig Vizepräsident der Kommission ist und den Vorsitz des Rates für Auswärtige Angelegenheiten innehat. Es wird befürchtet, dass eine Intergouvernementalisierung der GHP sowie eine Dominanz der Gemeinsamen Außen- und Sicherheitspolitik (GASP) über die GHP die Folge der Multifunktionalität dieses Amtes sein könne. Dieser Befürchtung konnte Dederer jedoch nicht zustimmen, da er den geltenden primärrechtlichen Verträgen keine Dominanz des einen über den anderen Politikbereich entnehmen konnte. Auch die Mitspracherechte des Parlaments dürften nach Dederer einer Intergouvernementalisierung der GHP entgegenstehen. In der Vorhand befinde sich der Hohe Vertreter lediglich dann, wenn sich der Vertragsgegenstand mindestens in der Hauptsache auf die GASP beziehe. Denn dann liege die Initiative zu Vertragsverhandlungen von vornherein allein beim Hohen Vertreter. Darüber hinaus könne die Kommission nach Art. 5 Abs. 3 UAbs. 2 des Ratsbeschlusses über die Organisation und die Arbeitsweise des Europäischen Auswärtigen Dienstes $(\mathrm{EAD})^{16}$ den Delegationen der Union Weisung erteilen, obgleich die Delegationen zum EAD und damit zum Hohen Vertreter gehören. Wie geschmeidig sich die GHP künftig ins Werk setzen lassen werde, hänge jedoch naturgemäß auch von den Persönlichkeiten der Akteure ab.

Im Anschluss sprach Christoph Vedder zunächst zur neuen Rolle einer Außenpolitik der Union in einer globalisierten Welt. Dann ging er auf die außenpolitischen Ziele ein, indem er zwischen speziellen Zielen der Handelspolitik in Art. 206 AEUV, der Einbettung der Handelspolitik in die generellen Ziele der AuBenpolitik über Art. 205, 207 Abs. 1 S. 2 AEUV i.V.m. Art. 21 EUV, der außenpolitischen Einbettung der internen Politiken nach Art. 21 Abs. 3 UAbs. 1 EUV und der Kohärenz sowohl zwischen den Außenpolitiken als auch zwischen externen und internen Politiken nach Art. 21 Abs. 3 EUV unterschied.
Nach einem Aufzeigen dieser Rahmenbedingungen benannte er die zwölf insbesondere in Art. 21 EUV genannten handels- und außenpolitischen Zielsetzungen der Union, die von der GATT- beziehungsweise WTO-Konformität der Handelspolitik über eine Integration aller Länder in die Weltwirtschaft, sicherheitspolitische Interessen, Menschenrechte, Demokratie und Rechtsstaatlichkeit, Achtung des Völkerrechts bis hin zu Nachhaltigkeit, Umweltschutz und, good governance' reichen. Echte Sanktionsmöglichkeiten im Falle eines Verstoßes ließen sich zwar grundsätzlich in den Handelsabkommen selbst beschließen. Da dies aber eher unwahrscheinlich sei, könne zumindest seitens der Union auch an einseitige Maßnahmen nach Art. 207 Abs. 2 AEUV gedacht werden. Der Kommentator und Leiter des Panels Horst Günter Krenzler führte an, dass eine Werteorientierung auch in der Handelspolitik heute nach dem primärrechtlichen Rahmen ein Muss geworden sei, dies sei in dieser Rigorosität bei keiner anderen Handelsmacht der Welt zu beobachten.

Die gemeinsame Handelspolitik als unionale Investitions(schutz)politik

Zunächst führte Markus Burgstaller zur Bedeutung und den Zielen des internationalen Investitionsschutzrechtes im Kontext der künftigen Unionskompetenzen einige Zahlen an. So könne ein Wachstumsfortschritt von Auslandsinvestitionen von 100 Milliarden US-Dollar im Jahr 1980 auf 1,4 Billionen USDollar im Jahr 2000 verzeichnet werden. Nach einem Wachstumsschwund aufgrund der Wirtschaftskrise in den Jahren 2007-2009 werde für das Jahr 2010 wieder ein Anstieg auf bis zu 1,2 Billionen US-Dollar erwartet. Seit den 1990er Jahren sei eine Verdichtung der Kodifikation des internationalen Investitionsschutzrechts durch die wachsende Zahl völkerrechtlicher bilateraler Investitionsschutzabkommen (BITs) zu beobachten. Der Anstieg von Streitbeilegungsentscheidungen

16 Amtsblatt der EU, Nr. L 201 vom 3. August 2010, S. 30-40. 
führe zu einer zunehmenden Konkretisierung der Schutzbestimmungen und damit auch zu größerem Vertrauen bei Investoren. Die Gesamtzahl der weltweit abgeschlossenen BITs sei in der ersten Jahreshälfte 2009 auf 2.792 Abkommen angestiegen, wobei in mehr als 1.500 BITs die Mitgliedstaaten der Europäischen Union involviert seien. Deutschland sei mit 137 in Kraft getretenen Abkommen Weltspitze, gefolgt von China, der Schweiz und dem Vereinigten Königreich. Als Ziele des internationalen Investitionsschutzrechts könne die Bildung von Vertrauensschutz seitens der Investoren durch die Vereinbarung materiellrechtlicher Schutzstandards und prozessrechtlicher Garantien zu deren Durchsetzung genannt werden. Damit tragen BITs zum Wirtschaftswachstum und zur positiven Entwicklung am Arbeitsmarkt bei.

Steffen Hindelang zeichnete die Kompetenzlage vor Inkrafttreten des Vertrags von Lissabon auf und wies insbesondere darauf hin, dass bereits bislang für Portfolioinvestitionen eine EG-Kompetenz aus den Vorschriften über die Kapitalverkehrsfreiheit entnommen wurde, genauer aus dem ehemaligen Art. 57 Abs. 2 EGV (jetzt Art. 64 Abs. 2 AEUV). Strittig war bislang, ob Direktinvestitionen eher unter die Niederlassungs- oder auch unter die Kapitalverkehrsfreiheit zu subsumieren seien, da grundsätzlich beide Aspekte betroffen seien. Hindelang skizzierte die hierzu ergangene uneinheitliche Rechtsprechung des EuGH. Er schloss seine Ausführungen mit dem Hinweis, dass die neue Unionskompetenz aus Art. 207 Abs. 1 AEUV allein Direktinvestitionen erfasse, sodass für Portfolioinvestitionen weiterhin die implizite Kompetenz aus der Kapitalverkehrsfreiheit fruchtbar zu machen sei. Unsicher sei, ob diese implizite Kompetenz auch den Streitbeilegungsmechanismus erfassen könne.

Als weiterer Referent sprach Nils Weith zunächst zur rechts- und wirtschaftspolitischen
Bedeutung der Investitionsförderungs- und -schutzverträge (IFV) aus deutscher Sicht, in dem er die Ziele deutscher Investitionspolitik, regionale Schwerpunkte und internationale Entwicklungen veranschaulichte. Er gab einen Überblick über den Bestand deutscher IFV und betonte, dass nur ganz wenige streitige Verfahren überhaupt jemals gegen Deutschland eingeleitet wurden. Er unterstrich die Vorreiterrolle, die Deutschland auf dem Parkett des Investitionsschutzrechts habe, seit es im Jahr 1959 den weltweit ersten bilateralen Investitionsschutzvertrag mit $\mathrm{Pa}$ kistan abgeschlossen hat. Darüber hinaus zeigte Weith die umfangreichen Garantien auf, die deutsche Investoren für Auslandsinvestitionen vom deutschen öffentlichen und privaten Sektor zu erhalten vermögen. Sodann ging der Referent auf die Ziele und Herausforderungen des Kompetenzübergangs auf die Union ein. Er stellte die Stärkung der Europäischen Union als Verhandlungspartner in den Vordergrund und betonte die Möglichkeit einer EU-weiten Harmonisierung des Schutzniveaus. Abschließend bewertete er den Verordnungsvorschlag der Kommission vom 7. Juli 2010 aus deutscher Sicht. ${ }^{17}$ Er unterstrich die Bedeutung der Bestandsgarantie in der Fortgeltung bestehender mitgliedstaatlicher Verträge als elementar wichtig für die Investoreninteressen und die Rechtssicherheit. Zudem begrüßte er eine Ermächtigung der Mitgliedstaaten für weitere Verhandlungen in der Übergangszeit. Er schloss seine Ausführungen mit der Feststellung, dass aus Sicht Deutschlands die Kompetenzen der Union aus Art. 207 AEUV sich nicht vollumfassend auf alle BIT-relevanten Sachmaterien erstrecken, so beispielsweise nicht auf Enteignungsfragen (mit Verweis auf Art. 345 AEUV Portfolioinvestitionen) und Streitschlichtungsklauseln. Insofern sei auch künftig von gemischten Abkommen unter Beteiligung der Mitgliedstaaten auszugehen.

17 Europäische Kommission: Vorschlag für eine Verordnung des Europäischen Parlaments und des Rates zur Einführung einer Übergangsregelung für bilaterale Investitionsabkommen zwischen Mitgliedstaaten und Drittländern, KOM (2010) 344. 
Jörn Griebel veranschaulichte zunächst das Schutzvakuum, das weltweit und auch in den Mitgliedstaaten der Europäischen Union derzeit im Investitionsrecht dadurch bestehe, dass eine Vielzahl an Staaten keine Investitionsabkommen geschlossen hätten oder darüber nachdachten, ihre Abkommen zu kündigen. Dann kritisierte er den Verordnungsentwurf der Kommission vom 7. Juli $2010,{ }^{18}$ in dem er insbesondere die dort verankerte Rücknahmemöglichkeit von Genehmigungen als nachteilig für Vertrauensschutz und Rechtssicherheit herausstellte. Des Weiteren kritisierte Griebel die Bestrebungen der Kommission, in künftigen Abkommen eine Verknüpfung von Handel und Investitionen vorzunehmen, als nachteilig für den Investitionsschutz. Er sah darin eine Absenkung des bisherigen Investitionsschutzniveaus. Handelsabkommen, die investitionsspezifische Zusatzklauseln enthielten, kämen nicht an den in bilateralen Investitionsschutzabkommen üblichen Schutzstandard heran. Dieser sei sehr viel ausgeprägter und lasse sich nicht mit einigen Zusatzklauseln bewirken. Nach Griebels Verständnis kommt der Europäischen Union als Wirtschaftsmacht die Möglichkeit $\mathrm{zu}$, den Inhalt internationaler Investitionsschutzabkommen maßgeblich $\mathrm{zu}$ beeinflussen. Aus der Sicht des Referenten ist es nicht nachvollziehbar, warum sie hiervon keinen Gebrauch macht. Griebel plädierte dafür, dass die Europäische Union ihren Einfluss nutzen und sich für ein multilaterales Investitionsschutzabkommen einsetzen solle.
Andreas $R$. Ziegler bewertete die im vierten Panel angeführten Aspekte aus Sicht der Schweiz als Nicht-EU-Mitgliedstaat. Er stellte den rasanten Bedeutungszuwachs internationaler Investitionsabkommen dar. Vor etwa zehn Jahren seien Investitionsschutzkapitel in völkerrechtlichen Lehrbüchern noch eine Neuheit gewesen. Mittlerweile sei der Bereich aus dem Wirtschaftsvölkerrecht nicht mehr wegzudenken. $\mathrm{Zu}$ dem von Griebel vorgeschlagenen Ansatz eines multilateralen $\mathrm{Ab}$ kommens führte Ziegler das Beispiel der vier Staaten der European Free Trade Association an. Diese seien vor einigen Jahren beim Versuch, ein gemeinsames Investitionskapitel zu verhandeln, an den widerstreitenden Interessen insbesondere Norwegens und der Schweiz gescheitert. Als weiteres negatives Beispiel führte er das Multilaterale Abkommen über Investitionen (MAI) an, das von der Organisation für wirtschaftliche Zusammenarbeit und Entwicklung (OECD) ausgearbeitet wurde und ein Vertragswerk zwischen transnationalen Konzernen, OECD-Staaten und der Europäischen Union hätte werden sollen, das jedoch nie in Kraft getreten ist. Als positive Beispiele gelungener multilateraler Übereinkommen in jüngerer Zeit stellte Ziegler das geplante Anti-Counterfeiting-Trade-Agreement gegen Produktpiraterie und Urheberrechtsverletzungen vor. Auch der Energiecharta-Vertrag mit seinem umfangreichen Investitionsschutzkapitel könne als positives Beispiel eines multilateralen Vertrags herangezogen werden.

18 Ebenda. 